\title{
Plexiform Schwannoma in the Duodenum of a Horse with Recurrent Colic
} Stephanie Olchowski ${ }^{1}$, Arlin B Rogers ${ }^{2}$, Jeremiah Lyons ${ }^{2}$ and Melissa R Mazan ${ }^{1^{*}}$

${ }^{1}$ Department of Clinical Sciences, Cummings School of Veterinary Medicine, Tufts University, 200 Westboro Road, North Grafton, MA 01536, USA ${ }^{2}$ Department of Biomedical Sciences, Cummings School of Veterinary Medicine, Tufts University, 200 Westboro Road, North Grafton, MA 01536, USA

*Corresponding author: Melissa R Mazan, Tufts Cummings School of Veterinary Medicine, 200 Westboro Road, North Grafton, MA 01536, USA, Tel: 508-839-5395; Email: melissa.mazan@tufts.edu

Received date: Oct 17, 2015; Accepted date: Dec 09, 2015; Published date: Dec 09, 2015

Copyright: (c) 2015 Olchowski S, et al. This is an open-access article distributed under the terms of the Creative Commons Attribution License, which permits unrestricted use, distribution, and reproduction in any medium, provided the original author and source are credited.

\begin{abstract}
Background: Intestinal neoplasia is an uncommon cause of colic in the horse. Accurate clinical diagnosis is necessary to direct rationale intervention because equine intestinal neoplasms have widely varying prognoses and treatment requirements.

Case Presentation: A 23-year-old Missouri Foxtrotter gelding presented with a history of mild recurrent colic. Physical examination, abdominal ultrasound, and cytologic evaluation of peritoneal fluid were unremarkable. Upper gastrointestinal endoscopy revealed multiple tan raised masses in the duodenal mucosa. Histopathology demonstrated a well demarcated, multicentric, intramucosal spindle cell neoplasm arranged in an Antoni A pattern with no evident mitotic figures or necrosis. Immunohistochemistry was negative for desmin, smooth muscle actin, and the gastrointestinal stromal tumor (GIST) marker CD117 (c-Kit). Tumor cells were uniformly positive for neuronal specific enolase and S-100. Based on distribution, morphology and immunohistochemical markers, the tumor was diagnosed as benign plexiform Schwannoma. The horse responded well to conservative symptomatic treatment.

Conclusion: Although uncommon, spindle cell tumors should be considered in cases of recurrent equine colic after more common causes have been excluded. Immunohistochemistry is necessary to definitively diagnose intestinal mesenchymal and neuroectodermal tumors. Benign tumors such as Schwannoma, neurofibroma and lowgrade GIST carry a good prognosis.
\end{abstract}

Keywords: Equine; Neoplasia; Schwannoma; Colic; Gastrointestinal; Immunohistochemistry; Gastroscopy

\section{Abbreviations:}

GIST: Gastrointestinal Stromal Tumor; PNST: Peripheral Nerve Sheath Tumor; IHC: Immunohistochemistry; SMA: Smooth Muscle Actin; NSE: Neuron-Specific Enolase

\section{Introduction}

Intestinal neoplasia is rare in the horse and is usually diagnosed postmortem. Lymphoma is the most common alimentary neoplasm followed by adenoma/adenocarcinoma, leiomyoma/leiomyosarcoma, gastrointestinal stromal tumor (GIST) and peripheral nerve sheath tumor (PNST) [1]. Immunohistochemistry with or without ultrastructural confirmation is required to distinguish spindle cell tumors $[2,3]$. Here we report a case of benign plexiform Schwannoma in the duodenum of a horse with recurrent colic. To our knowledge this is the first documentation of equine foregut Schwannoma, and the only description of an endoscopically-diagnosed neuroectodermal intestinal tumor with successful clinical management in this species.

\section{Case Presentation}

A 23-year old Missouri Foxtrotter gelding was presented to the Cummings School of Veterinary Medicine, Hospital for Large Animals, with a three-day history of colic and decreased appetite. Previous bouts of spontaneously resolving colic also were reported. Vital parameters were within normal limits while gastrointestinal sounds were decreased. Palpation per rectum and percutaneous abdominal ultrasonographic examination were unremarkable. No net reflux was obtained upon on nasogastric intubation. Cytology and biochemical analysis of peritoneal fluid showed no abnormalities. In contrast, endoscopy revealed extensive ulceration of the glandular stomach and multiple raised tan masses in the proximal duodenum (Figure 1). Multiple mucosal pinch biopsies were collected. Histopathology revealed a multicentric, sharply demarcated spindle cell neoplasm within the lamina propria displacing preexisting glands. Tumor cells were elongate, fusiform and arranged in tight fascicles and whorls in an Antoni A-type pattern. There was little cellular or nuclear pleomorphism, and no mitotic figures or areas of necrosis. Immunohistochemistry (IHC) was negative for the smooth muscle markers desmin and smooth muscle actin (SMA). Gastrointestinal stromal tumor (GIST) was ruled out by negative staining for CD117 (cKit) (Figure 2). Neuroectodermal markers neuron-specific enolase (NSE) and S-100 were uniformly expressed throughout the tumor. Based on morphology and IHC, the tumor was diagnosed as benign plexiform Schwannoma. Following gastroscopy the patient became increasingly uncomfortable and began producing gastric reflux. The stomach was periodically decompressed and supportive care was initiated. A 28-day course of the proton-pump inhibitor omeprazole $\left(\right.$ Gastrogard $\left.{ }^{\oplus}\right)$ was initiated to alleviate gastric ulceration. The patient responded well to medical management and was reintroduced to food over the next few days. No adverse effects have been reported since 
Page 2 of 3

patient discharge apart from occasional bouts of colic that respond well to home-based medical management.

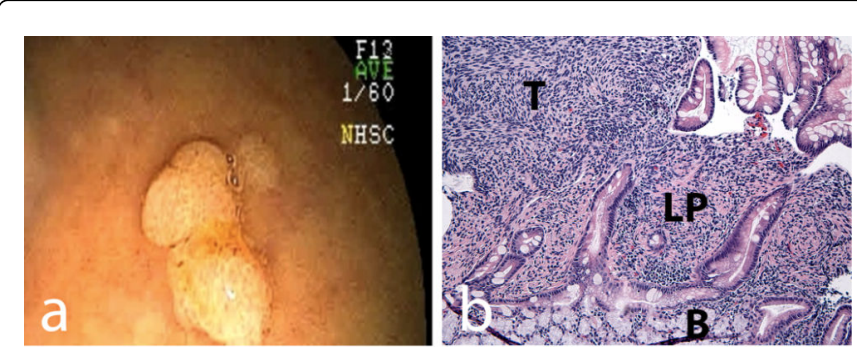

Figure 1: (a) Endoscopic image revealing one of several irregular, sessile, tan intramucosal masses in the proximal duodenum. (b) Histology demonstrates a spindle cell tumor $(\mathrm{T})$ in the duodenal mucosa displacing pre-existing glands; adjacent lamina propria (LP) exhibits moderate mixed inflammation; pale flocculent Brünner's glands (B) lie below; H\&E, 200X.

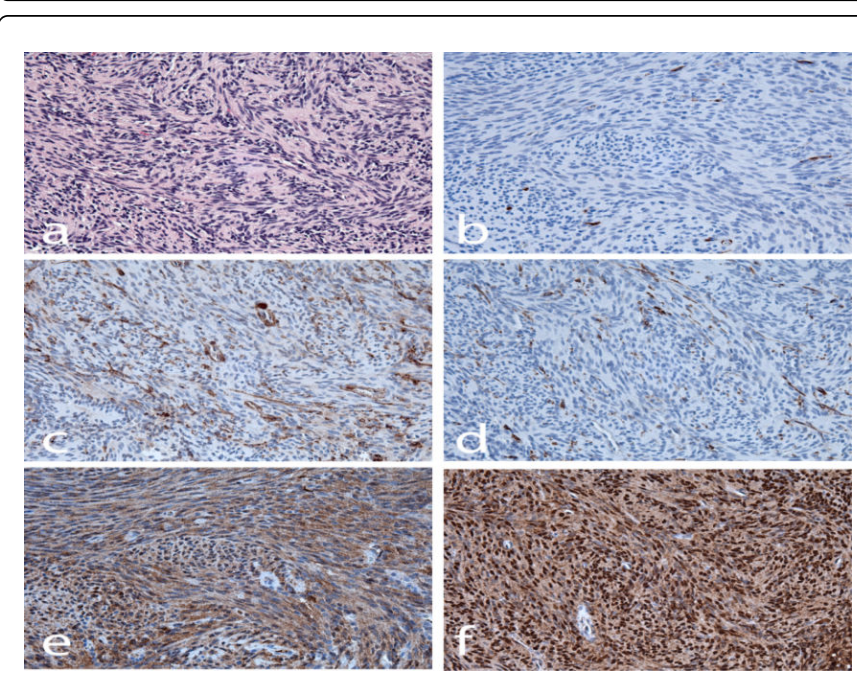

Figure 2: (a) Higher magnification H\&E stain of tumor demonstrates elongate spindle cells tightly arranged in fascicles and whorls in an Antoni A-type pattern. (b) CD117 (c-Kit) IHC is negative except for scattered infiltrating mast cells. (c) Smooth muscle actin highlights rich intratumoral capillary network and thin supporting stromal branches; tumor cells are negative. (d) Desmin is negative except for some supporting stromal cells. (e) Neuron-specific enolase uniformly labels tumor cells predominantly within the cytoplasm. (f) S-100 uniformly labels tumor nuclei intensely and cytoplasm moderately. All magnifications $400 \mathrm{X}$.

\section{Discussion}

Intestinal neoplasia is rare in the horse, and usually is identified at autopsy. Antemortem diagnosis is crucial to diagnose and appropriately manage horses with intestinal tumors. In a retrospective study of 34 horses with postmortem-verified intestinal cancer, a definitive antemortem diagnosis was not reached in $62 \%$ of the cases [1]. In the same study, abdominal ultrasound yielded high sensitivity but low specificity. Examination of peritoneal fluid can aid in the diagnosis of lymphoma, but has limited utility for non-lymphoid intestinal tumors [1]. Definitive diagnosis requires histopathology and, in the case of spindle cell neoplasms, immunohistochemistry to stratify tumors by cell of origin [1-5]. In our investigation, adenocarcinoma and neuroendocrine tumor/carcinoid were ruled out by the presence of elongate spindle cells tightly arranged in fascicles and whorls in an Antoni A-type pattern. GIST was discounted due to the lack of reactivity for CD117/c-Kit [6,7]. Therefore, the primary ruleouts were neurofibroma, gangliocytic paraganglioma, and Schwannoma. Neurofibroma consists of a loosely arranged mix of mesenchymal and neural-crest -derived cells, whereas gangliocytic paraganglioma includes fibroblastic, gangliocytic and neuroendocrine-type cells. The homogeneous pattern of the duodenal neoplasm in this case, combined with uniform staining for NSE and S-100 was consistent with Schwannoma. Because the neoplasm was multicentric but lacked cytologic features of malignancy, a final diagnosis of benign plexiform Schwannoma was assigned. Good therapeutic response to conservative medical management confirmed the benign nature of the disease [8-11].

\section{Conclusion}

Intestinal spindle cell neoplasms are uncommon causes of equine colic. Accurate diagnosis requires endoscopic biopsy and immunohistochemistry. If benign, cases might be amenable to medical management with a good prognosis.

\section{Acknowledgements}

The authors thank Francis Brown, Supervisor of the Cummings School Histopathology Laboratory, for expert histology and immunohistochemistry.

\section{References}

1. Taylor SD, Pusterla N, Vaughan B, Whitcomb MB, Wilson WD (2006) Intestinal neoplasia in horses. J Vet Intern Med 20: 1429-1436.

2. Del Piero F, Summers BA, Cummings JF, Mandelli G, Blomme EA (2001) Gastrointestinal stromal tumors in equids. Vet Pathol 38: 689-697.

3. Muravnick KB, Parente EJ, Del Piero F (2009) An atypical equine gastrointestinal stromal tumor. J Vet Diagn Invest 21: 387-390.

4. Hafner S, Harmon BG, King T (2001) Gastrointestinal stromal tumors of the equine cecum. Vet Pathol 38: 242-246.

5. Maas CP, ter Haar G, van der Gaag I, Kirpensteijn J (2007) Reclassification of small intestinal and cecal smooth muscle tumors in 72 dogs: clinical, histologic, and immunohistochemical evaluation. Vet Surg 36: $302-313$.

6. Towu E, Stanton M (2006) Gastrointestinal stromal tumour presenting with severe bleeding: a review of the molecular biology. Pediatr Surg Int 22: $462-464$

7. Terada T (2013) Smooth muscles and stem cells of embryonic guts express KIT, PDGFRRA, CD34 and many other stem cell antigens: suggestion that GIST arise from smooth muscles and gut stem cells. Int J Clin Exp Pathol 6: 1038-1045.

8. Pascoe PJ (1982) Colic in a mare caused by a colonic neurofibroma. Can Vet J 23: 24-27.

9. Schöniger S, Summers BA (2009) Localized, plexiform, diffuse, and other variants of neurofibroma in 12 dogs, 2 horses, and a chicken. Vet Pathol 46: 904-915.

10. Strubbe DT (2001) Periocular neurofibrosarcoma in a horse. Vet Ophthalmol 4: 237-241. 
Citation: Olchowski S, Rogers AB, Lyons J, Mazan MR (2015) Plexiform Schwannoma in the Duodenum of a Horse with Recurrent Colic. J Cytol Histol S3: 028. doi:10.4172/2157-7099.S3-028

Page 3 of 3

11. Kirchhof N, Scheidemann W, Baumgartner W (1996) Multiple peripheral nerve sheath tumors in the small intestine of a horse. Vet Pathol 33: 727-730.

This article was originally published in a special issue, entitled:

"Cytopathology", Edited by Borislav A. Alexiev Department of Pathology

University of Maryland Medical Center USA 